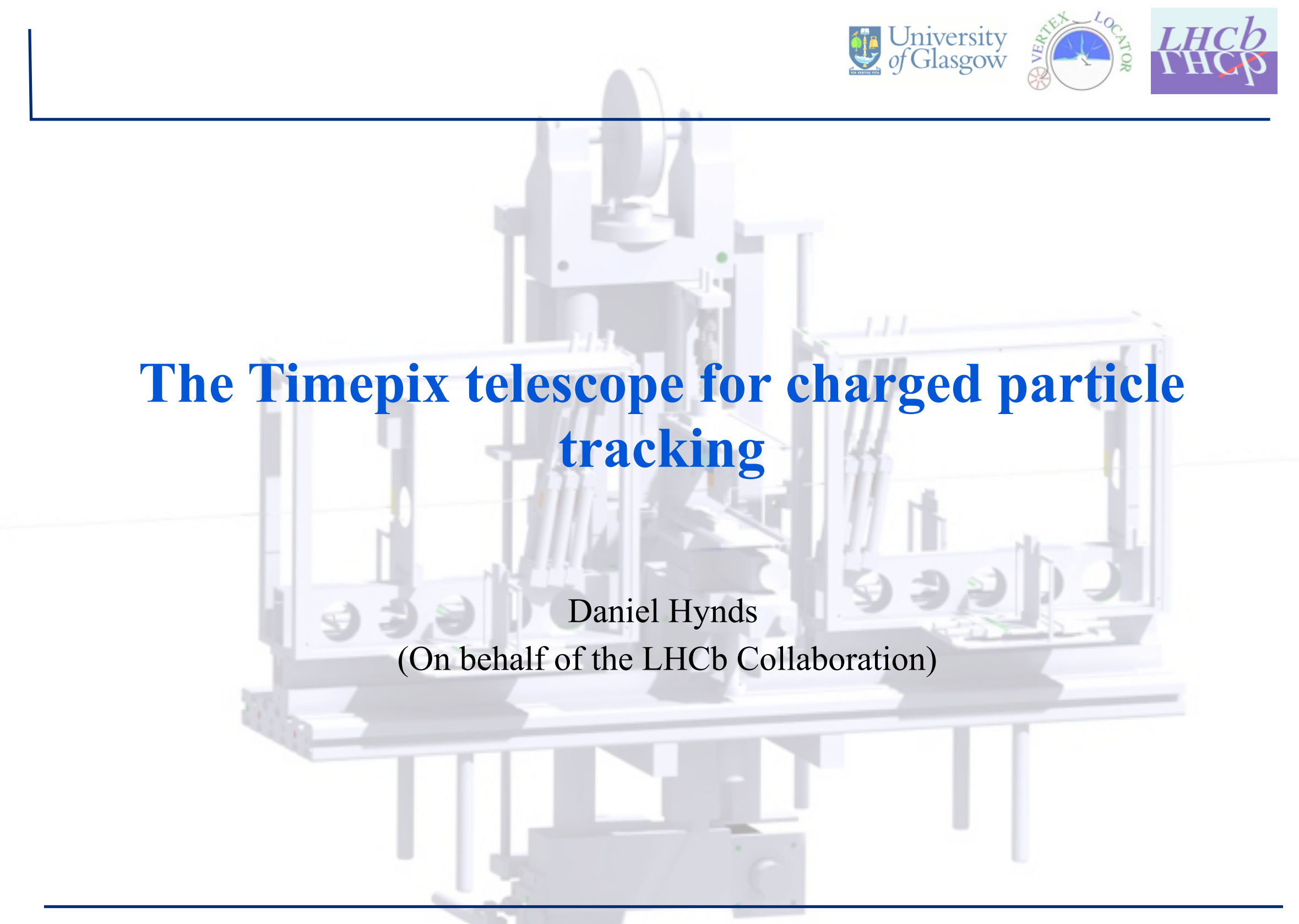




\section{Why bother...?}

- Telescope built primarily to qualify the TimePix chip as a viable option for the Velo Upgrade (see talk of P.Rodriguez)

- Collaboration between Medipix group and LHCb

- Extended under AIDA project to provide flexible, high precision, fast telescope to support generic detector R\&D (specifically including LHC-style readout)

- Telescope based in CERN SPS North Area (H8)
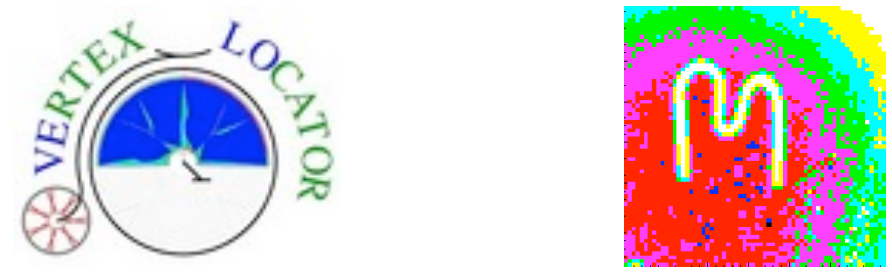

AIDA 


\section{TimePix chip}

- TimePix: $55 \mu \mathrm{m}$ pitch hybrid pixel chip

- Three modes of operation

- Medipix: the pixel records the number of times that the input charge was over threshold (photon counting)

Amplifier output (with threshold)

Medipix

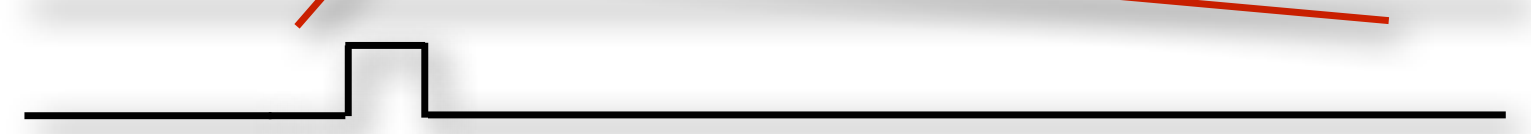




\section{TimePix chip}

- TimePix: $55 \mu \mathrm{m}$ pitch hybrid pixel chip

- Three modes of operation

- Medipix: the pixel records the number of times that the input charge was over threshold (photon counting)

- ToA (Time of Arrival): pixel starts counting with first hit, counts until shutter closes

Amplifier output (with threshold)

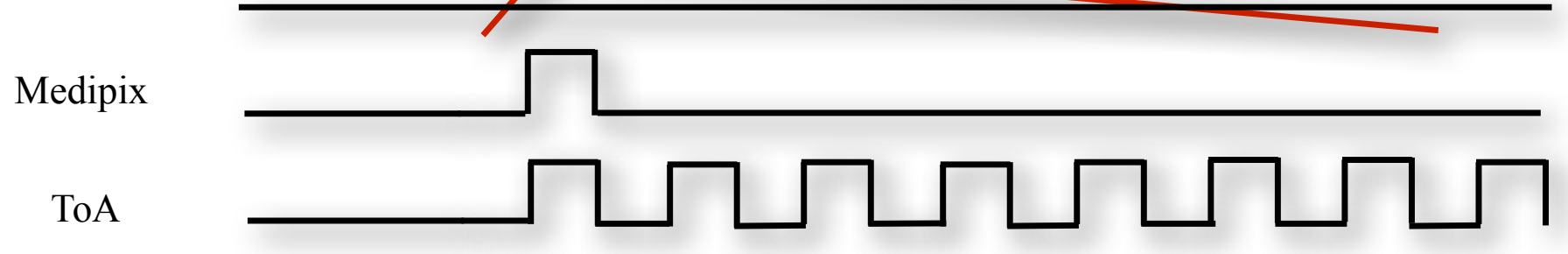




\section{TimePix chip}

- TimePix: $55 \mu \mathrm{m}$ pitch hybrid pixel chip

- Three modes of operation

- Medipix: the pixel records the number of times that the input charge was over threshold (photon counting)

- ToA (Time of Arrival): pixel starts counting with first hit, counts until shutter closes

- ToT (Time over Threshold): linear pixel discharge, time over threshold $\propto$ charge

Amplifier output (with threshold)

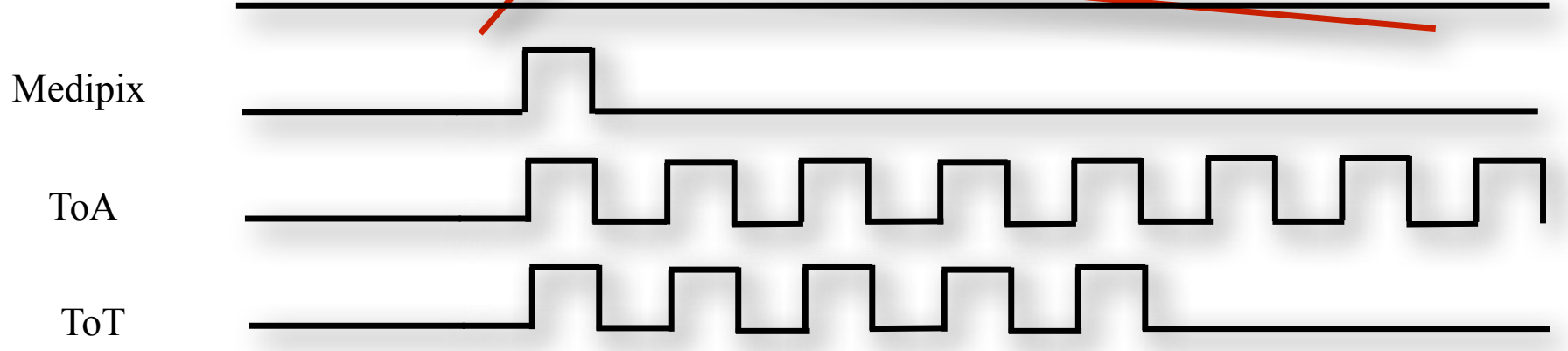




\section{Telescope setup}

- Readout system developed at Nikhef - RELAXD

- Whole telescope built for flexibility - remote movement of telescope ( $\mathrm{x}-\mathrm{y}$ for whole setup, $\mathrm{x}-\mathrm{y}-\theta$ for DUT) + DUT, mounting inside/upstream/downstream

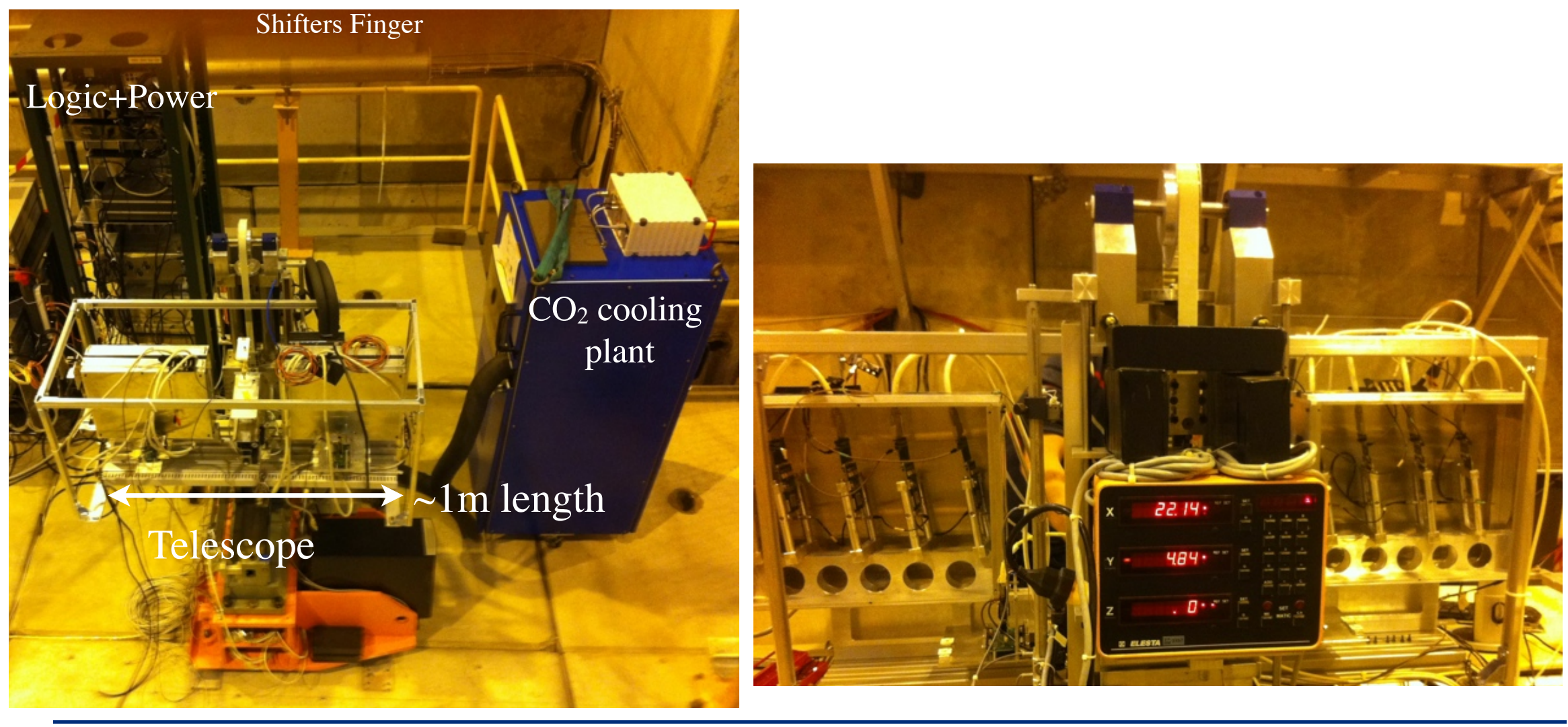




\section{Data taking}

- Data taking is shutter based

- Bursts of triggers then $\sim 10 \mathrm{~ms}$ readout dead time

- Each telescope frame contains many tracks - time matched offline to DUT data

- Max frame rate of $\sim 60 \mathrm{~Hz}$, with $10-200$ tracks/frame gives up to $\mathbf{1 2} \mathbf{k H z}$ track rate

- No hardware integration of the DUT. Data taking separate, only interaction between systems is trigger to and busy from DUT. Hardware timestamp required in DAQ

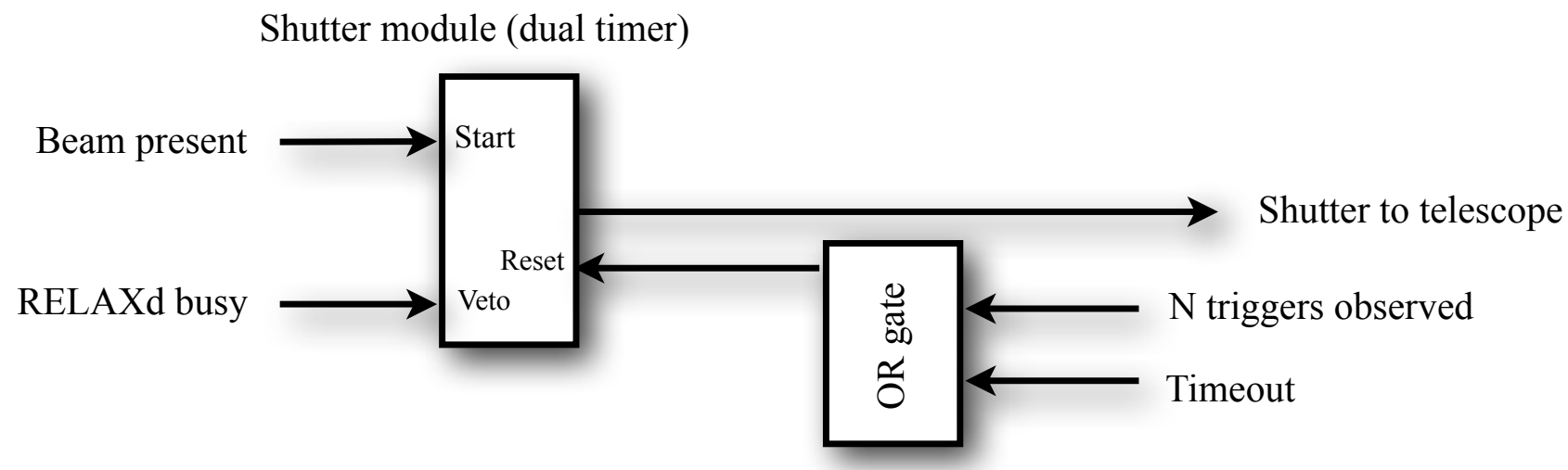




\section{External users}
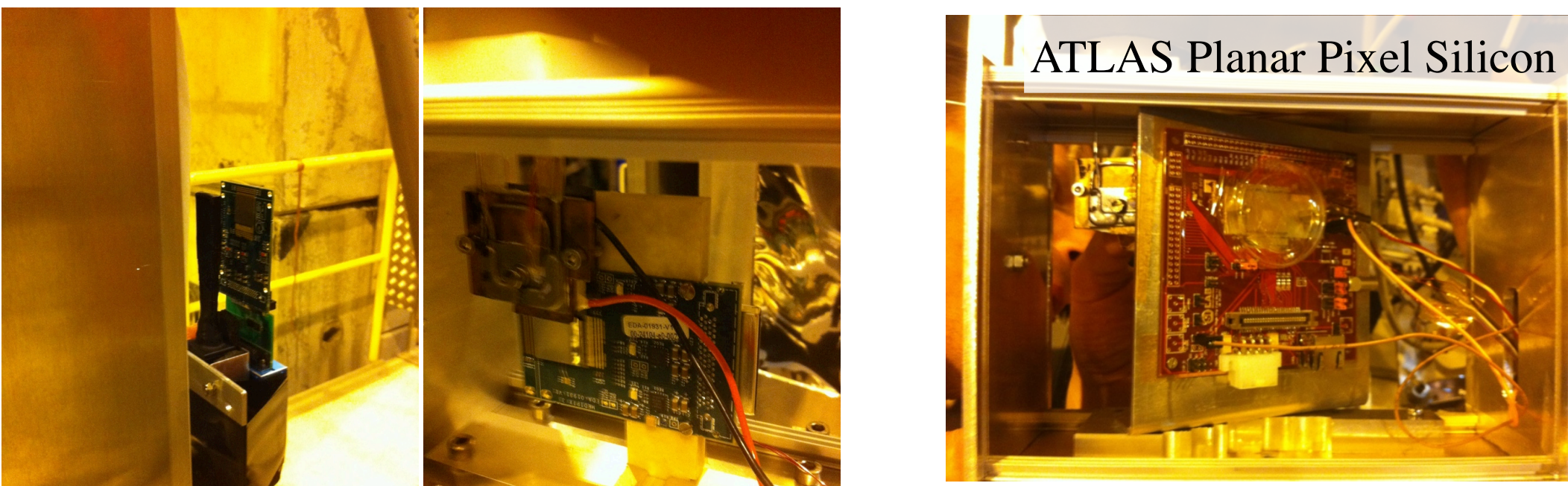

Medipix3 - PXI readout system

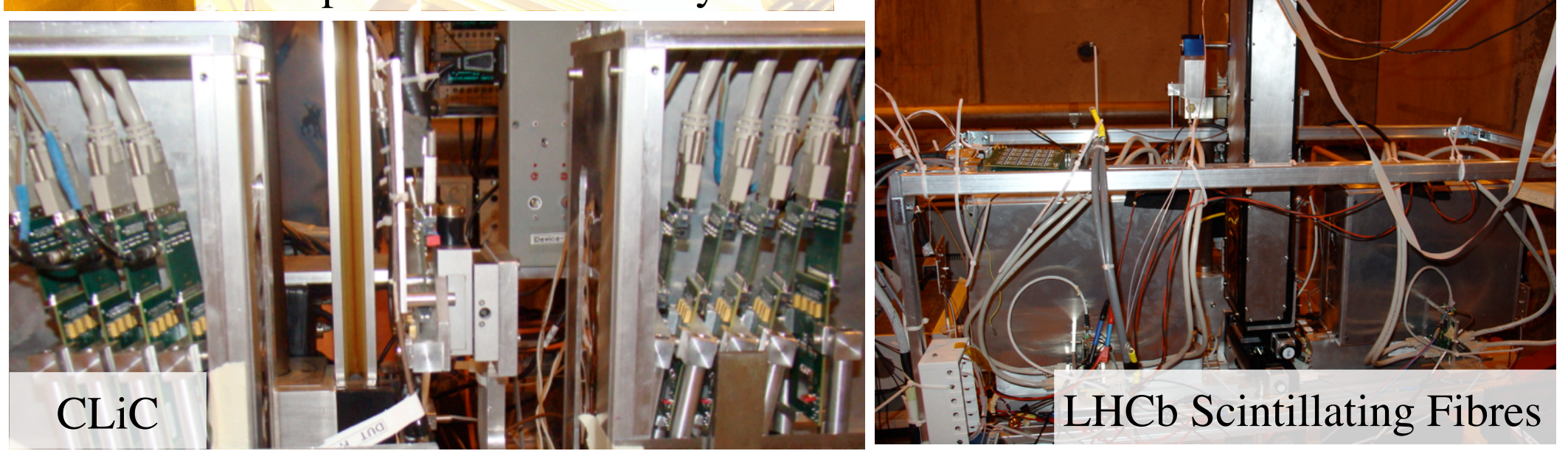




\section{Pattern recognition}

- Tracks are reconstructed using a straightforward extrapolation along z (beam) axis

- Extrapolate in z, look for clusters, fit prototrack, extrapolate, search...

- Low chip noise vital for clean reconstruction

z-axis extrapolation and KDTree search

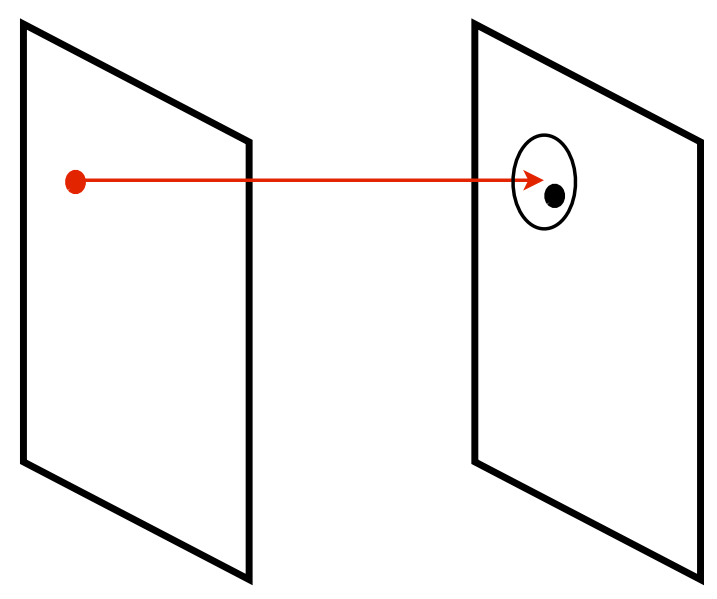




\section{Pattern recognition}

- Tracks are reconstructed using a straightforward extrapolation along z (beam) axis

- Extrapolate in z, look for clusters, fit prototrack, extrapolate, search...

- Low chip noise vital for clean reconstruction

track segment fit and extrapolation, followed by KDTree search

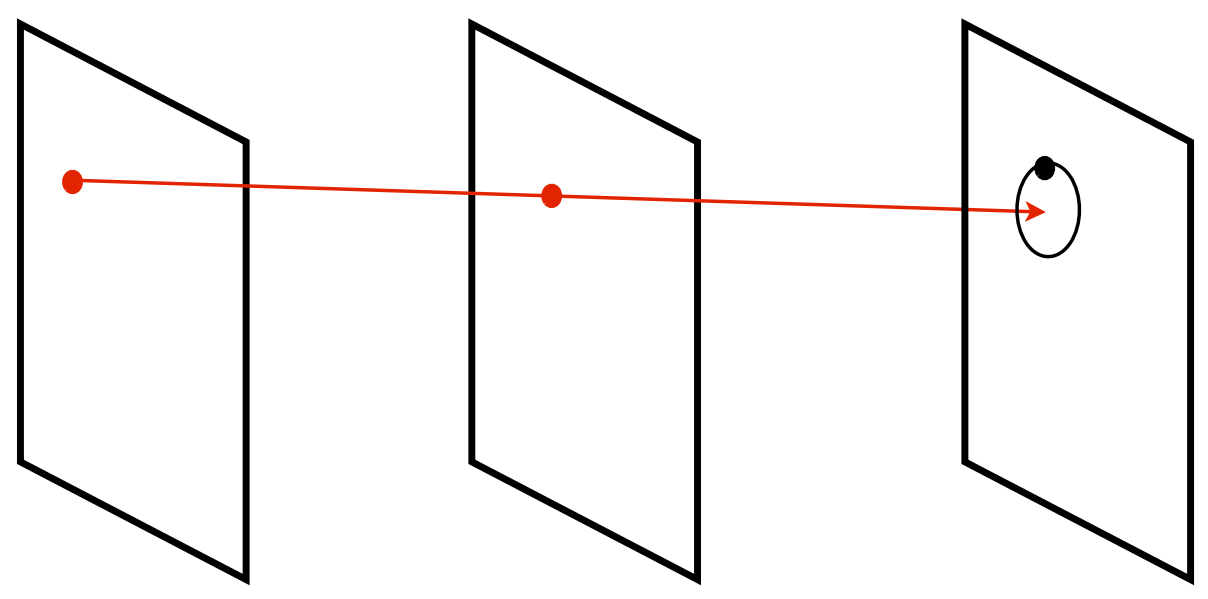




\section{Pattern recognition}

- Tracks are reconstructed using a straightforward extrapolation along z (beam) axis

- Extrapolate in z, look for clusters, fit prototrack, extrapolate, search...

- Low chip noise vital for clean reconstruction

track segment fit and extrapolation, followed by KDTree search

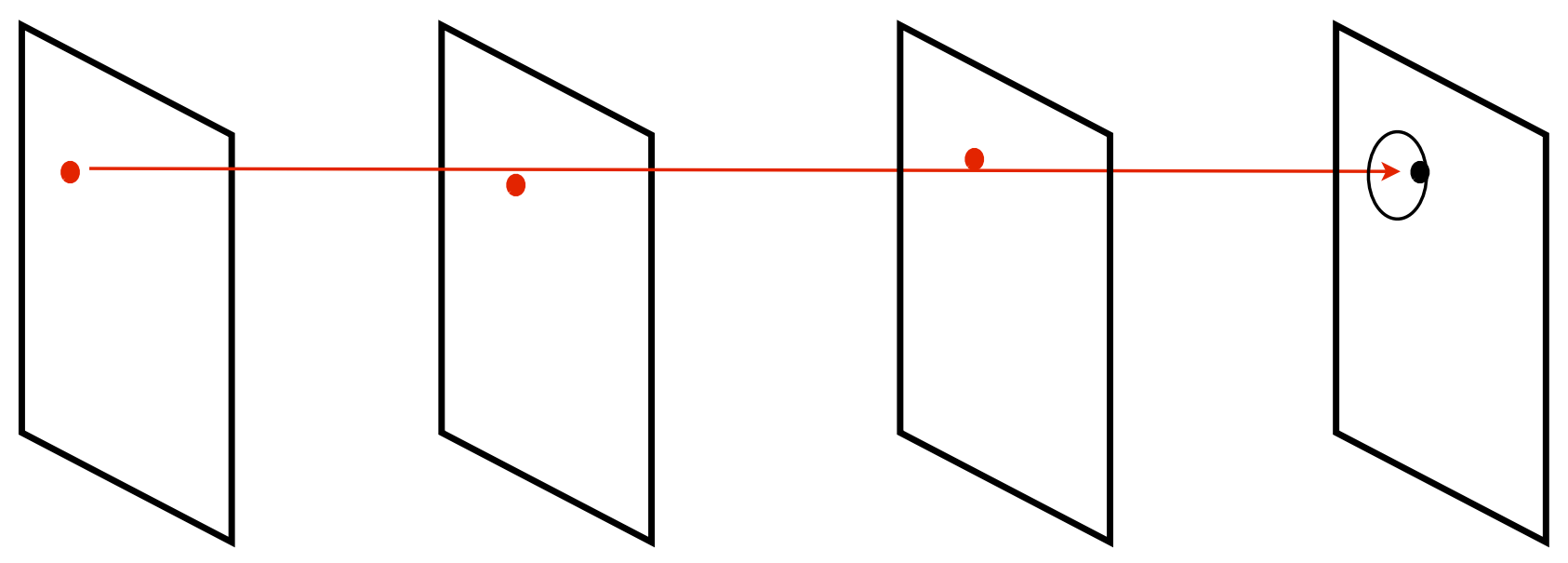




\section{Pattern recognition}

- Tracks are reconstructed using a straightforward extrapolation along z (beam) axis

- Extrapolate in z, look for clusters, fit prototrack, extrapolate, search...

- Low chip noise vital for clean reconstruction

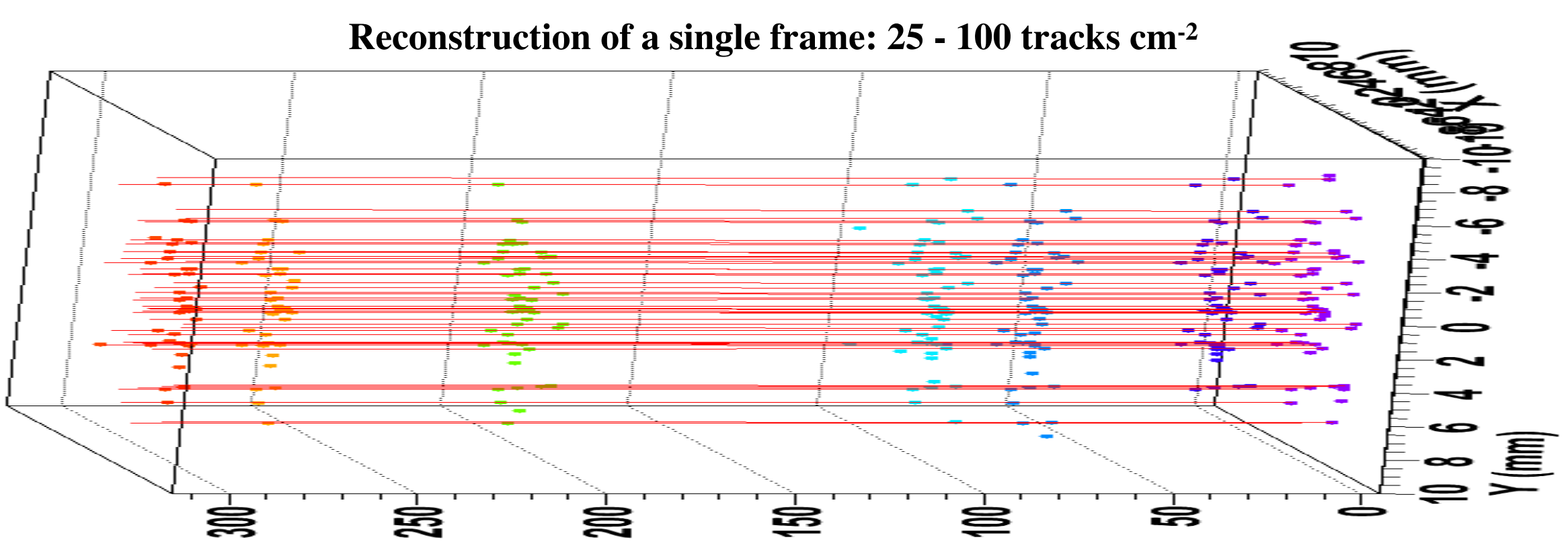




\section{Performance}

Single plane resolution versus angle

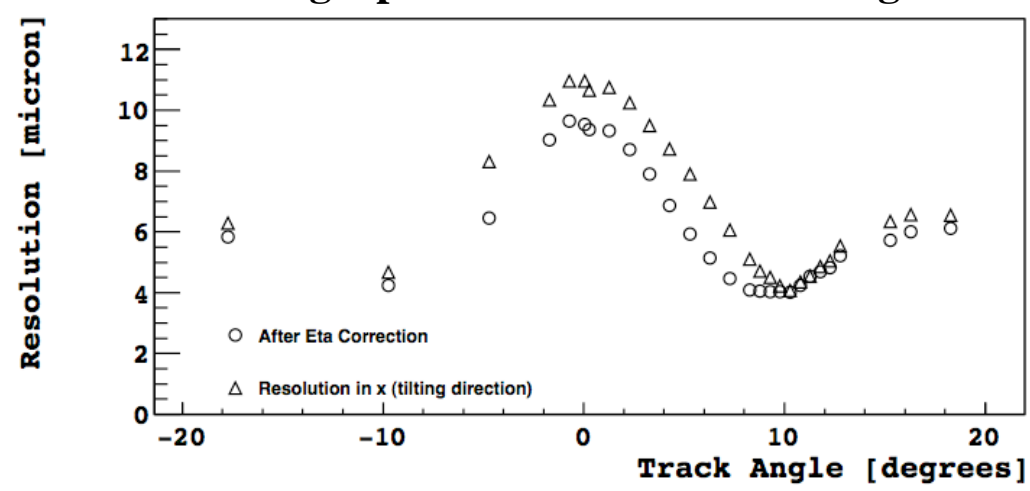

MC Determination of pointing resolution

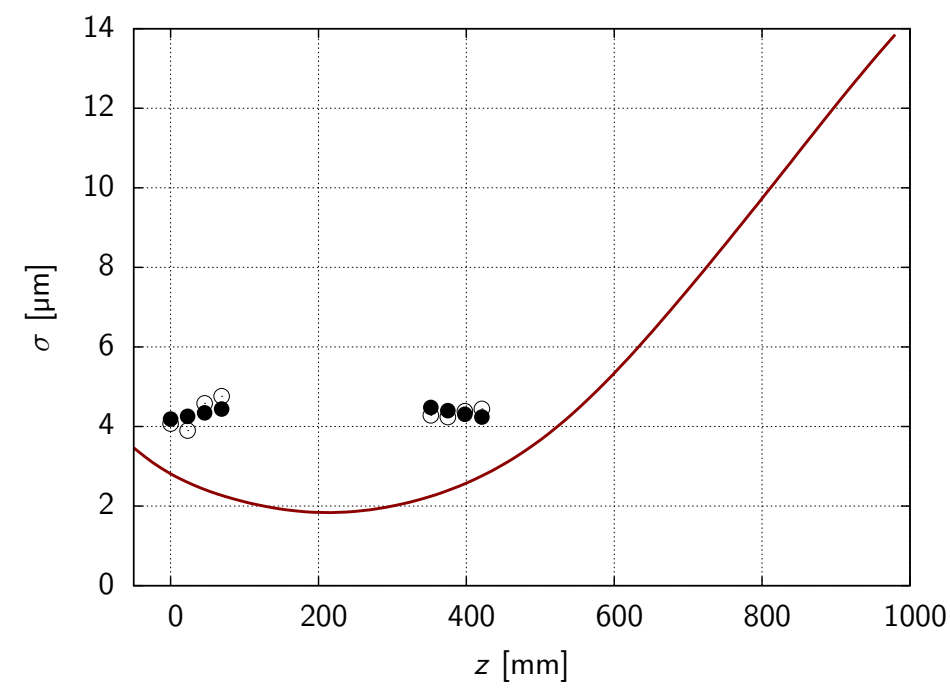

- $\quad$ Single chip extensively tested before building the telescope proper

- $\quad$ K.Akiba et al, Nucl. Instr. A 661 (2011) 31-49

- For $300 \mu \mathrm{m}$ sensor at moderate bias, best resolution of $\sim \mathbf{4} \boldsymbol{m}$ achieved

- $\quad$ From MC including scattering and single plane resolution, get $<\mathbf{2} \boldsymbol{\mu m}$ pointing resolution

Single plane performance plots
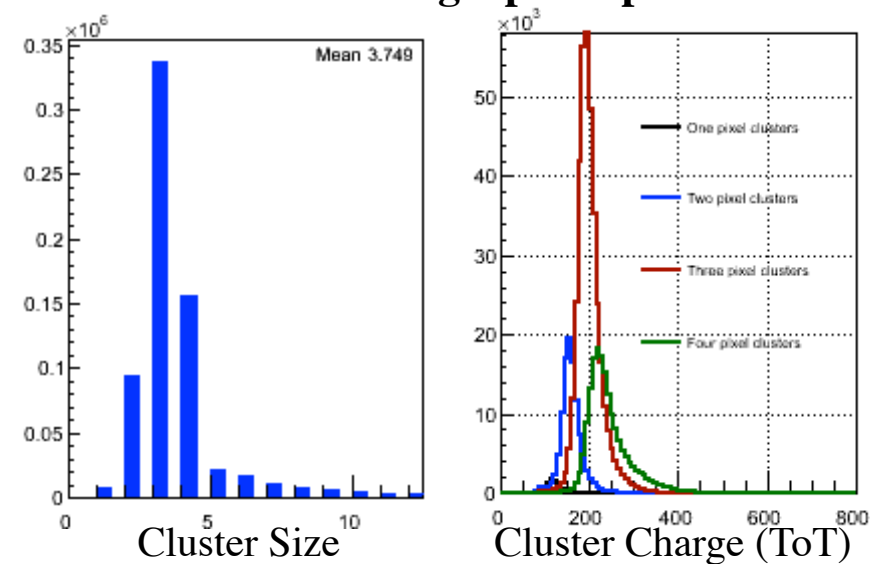


\section{Reconstruction efficiency}

- Detection efficiency of chips $>99 \%$ (as expected)

- Adding more tracks in the same area means distance between tracks scales as $1 /$ occupancy $^{2}$ )

- Inefficiencies in track reconstruction come (mainly) from

- Tracks being close to each other

- Multiple scattering

Tracks reconstructed v. tracks expected

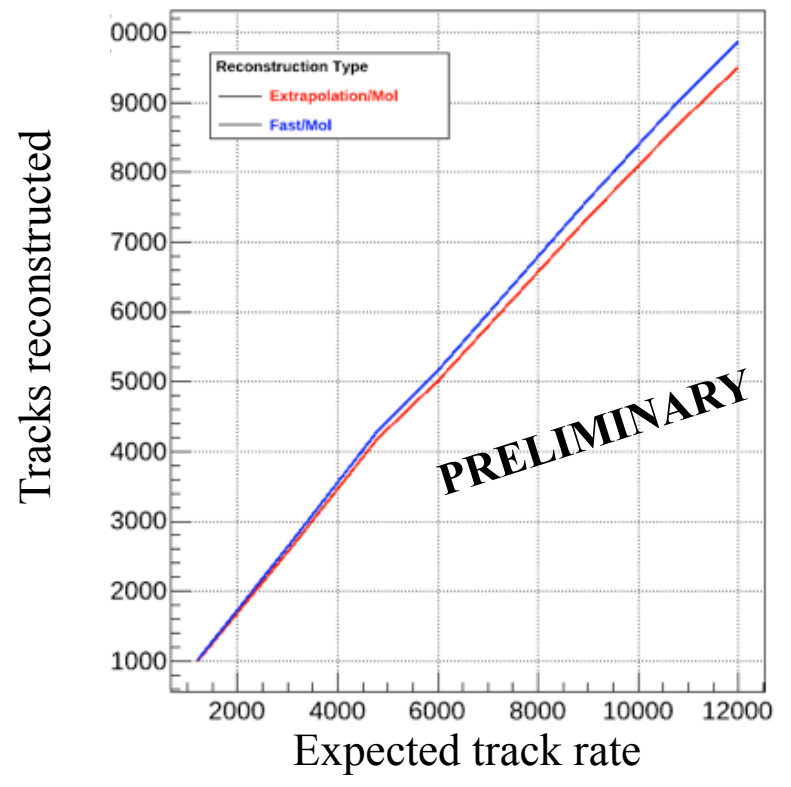

CPU for reconstruction / event v. occupancy

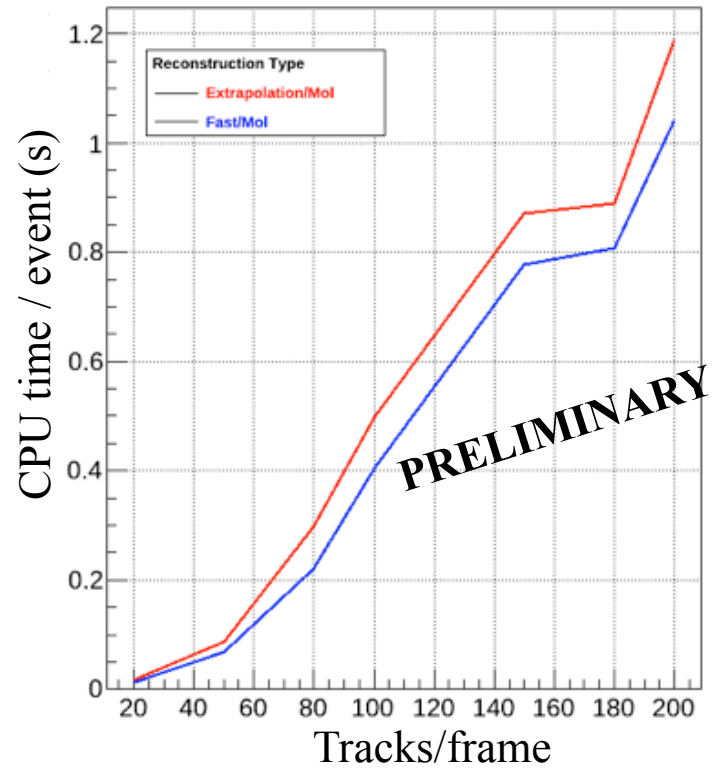




\section{Performance - results with dut}

Resolution v. angle for $2 \times 10^{15} 1 \mathrm{MeV}_{\text {eq }} \mathbf{c m}^{-2}$

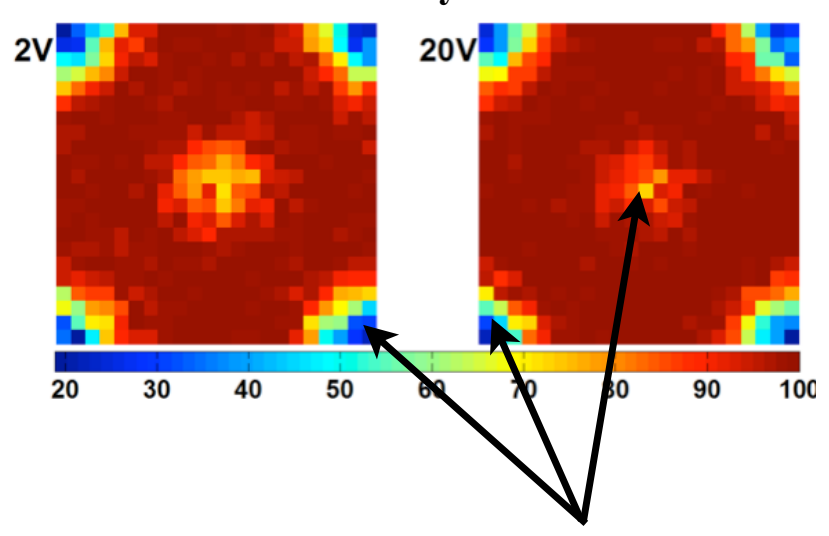

Column structure

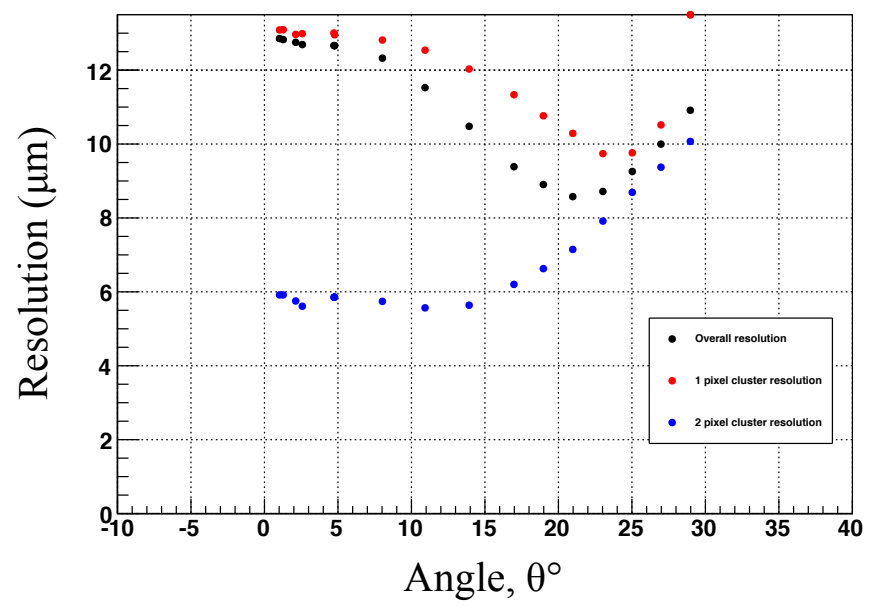

Charge sharing studies - location of n-pixel clusters across the unit cell
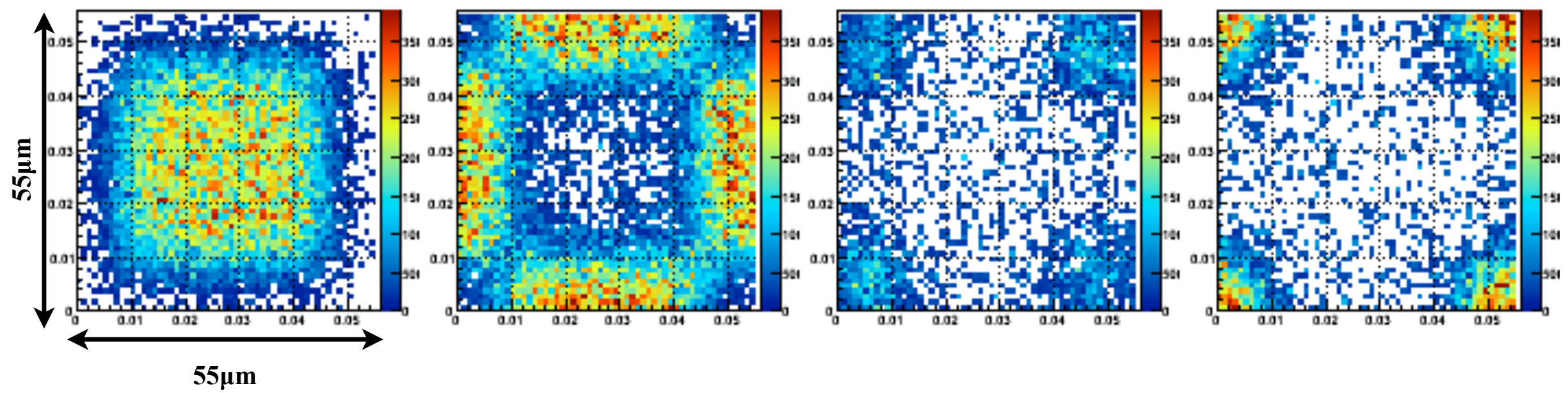


\section{The future - TimePix3}

- New chip from Medipix3 collaboration, submission end of 2012

- Testbed for several experiments interested in custom TimePix chips (LHCb, CLiC..)

- Frame based concept discarded in favour of high-rate data-driven readout

- Environments such as LHCb VELO upgrade require all data to be sent off-detector, for each 25ns bunch crossing $=>$ no time for triggers

- Data driven readout

- Will give huge advantages in terms of rate and event reconstruction

- Fast oscillator for timing measurements better than global clock frequency (1.6ns resolution)

- Simultaneous charge and time measurement

- 20 Mhits $\mathrm{cm}^{-2}$

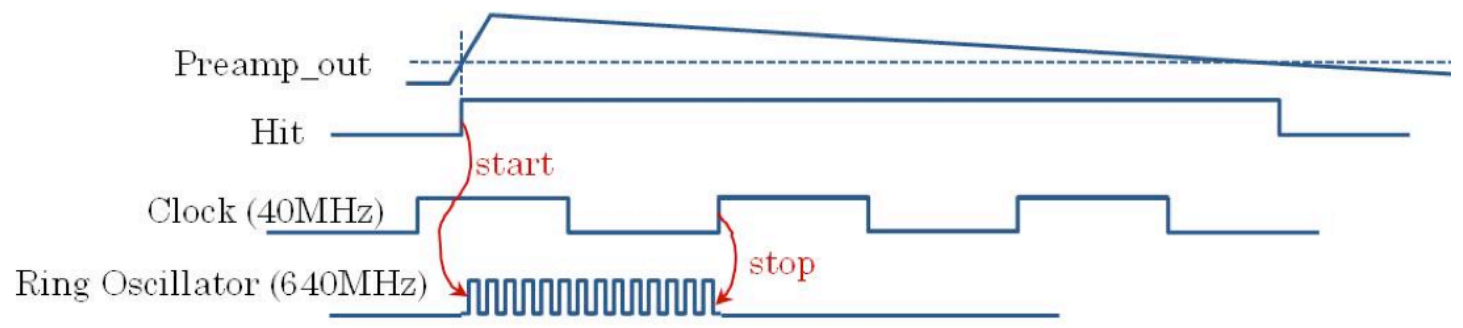




\section{Summary}

- TimePix telescope has been developed as a high-speed, high-precision tracking telescope

- Pointing resolutions of less than $\mathbf{2} \boldsymbol{\mu m}$ have been demonstrated, with $\mathbf{n s}$ time resolution, at average track rates between $3 \mathbf{k H z}$ and $\mathbf{1 2} \mathbf{k H z}$

- External device integration has been carried out under the philosophy of minimal hardware integration and time-matching of data offline - results with external devices have been shown (and presented elsewhere in this conference)

- An upgrade to TimePix 3 chips is expected once available in 2012

- Time tagging and data driven readout will lead to cleaner event reconstruction

- Data rates far higher than present will be achievable

- Eventually leading on to a fully pixel-VELO prototype...?

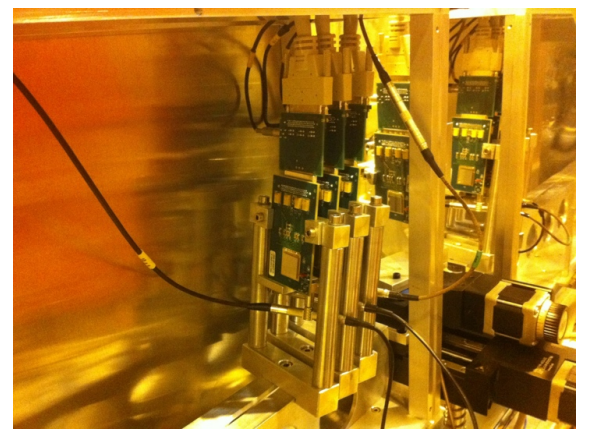




\section{BACKUP}




\section{Telescope setup}

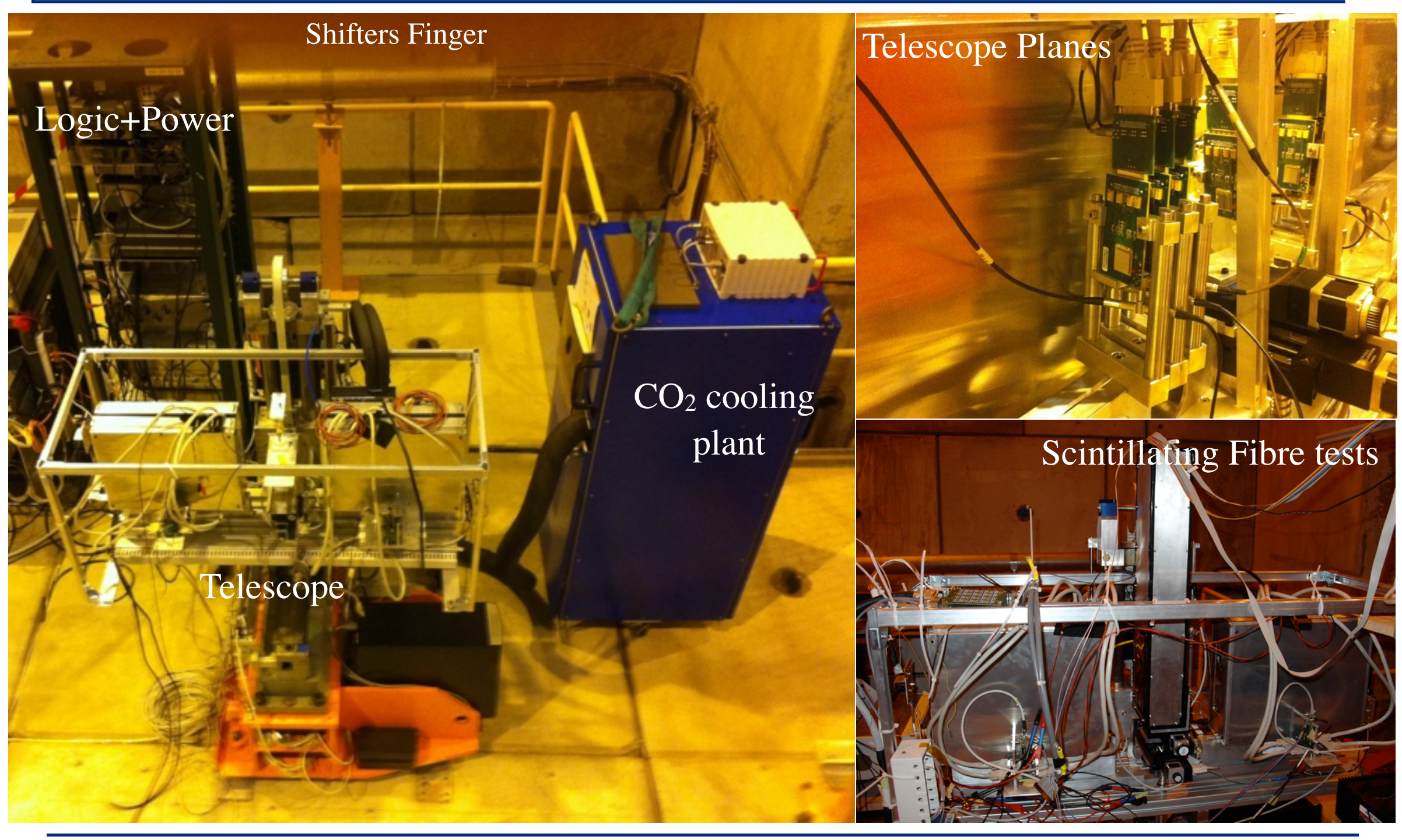




\section{External DUT integration}

- Ethos behind integration of devices has been driven by experience and cynicism

- There is always something new to test, that only arrived yesterday, and is read out with an entirely different set of hardware + software...

- Integration required to take data has been kept to a minimum

- For event-triggered devices only a trigger and busy signal are used

- A time stamp is required on the external device data, to match the data offline

- Timestamp should be implemented in hardware - O(100ns) sufficient

- Offline, each shutter looks like a barcode

- Look at trigger spacings in DUT and TDC

- Set strict requirements on matched fractions to avoid mis-matching ( $95 \%)$

Shutter (telescope continuously sensitive)

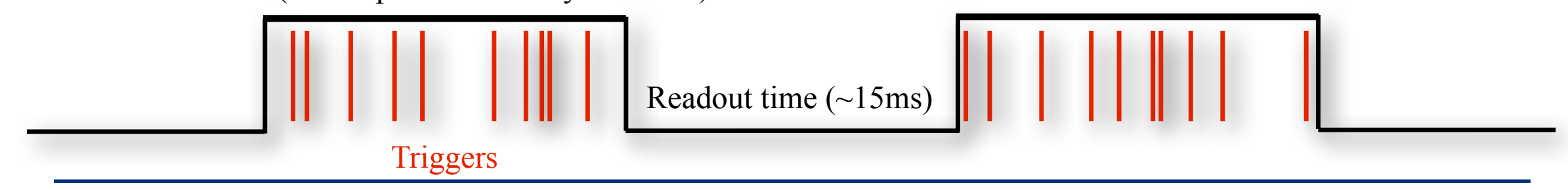




\section{Analysis Software}

- Analysis software based loosely on Gaudi framework

- All algorithms consist of 3 parts:

- Initialisation - histograms are initiated, variables set up, etc.

- Execution - this is the code which is run on each frame

- Finalisation - any actions which are required after running over all events

- Events (shutters) are analysed sequentially, with each event passing through the analysis stages requested

- Information passed between algorithms

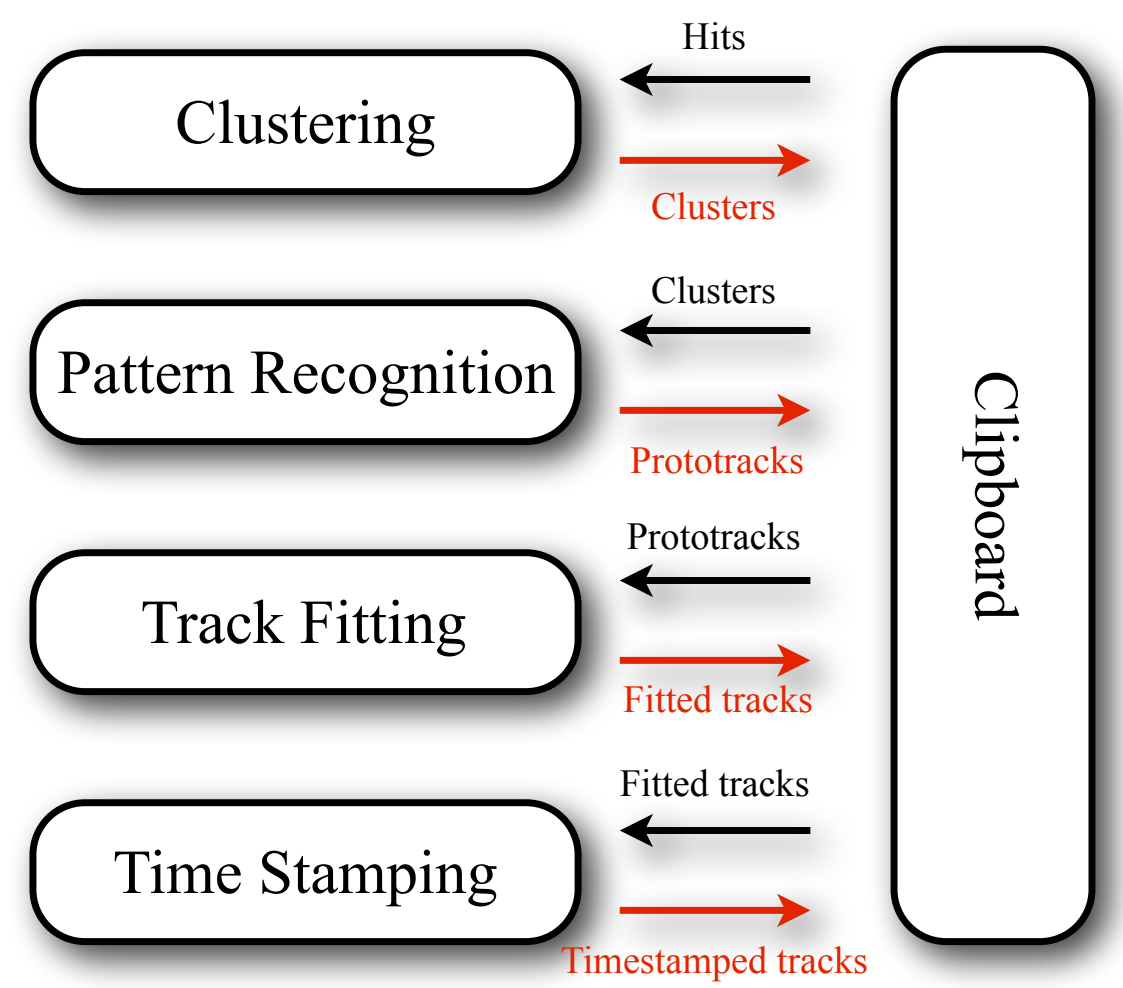
using global "clipboard" 


\section{Track time-stamping}

- Timing plane records time between particle arrival and shutter close

- Granularity of global clock - tuneable from $100 \mathrm{MHz}$ to $2.5 \mathrm{MHz}(\sim 300 \mathrm{kHz}$ if externally supplied)

- For practical data taking, this means tuning of parameters to beam conditions

- Granularity of ToA clock should be much less than time between triggers

- Max shutter length should then be $11800 \times$ clock period

- Number of triggers per frame should be between 10 and 200

- Tracks are reconstructed spatially, with last plane containing timing information instead of charge

- Track gains a timestamp with respect to the shutter close

- TDC records not only the trigger times, but shutter open and close

- TDC information used to extract "real" track time with resolution $\sim 1$ ns

Time between triggers (TDC)

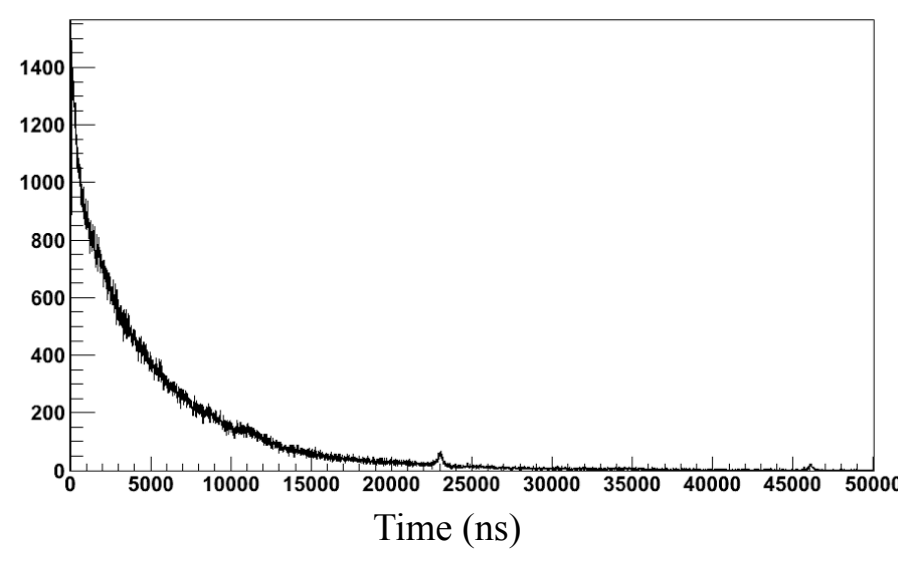




\section{The future - TimePix3}

- New chip from Medipix3 collaboration

- $\quad$ Expected submission end of 2012

- Testbed for several experiments interested in custom TimePix chips (LHCb, CLiC..)

- Frame based concept discarded in favour of high-rate data-driven readout

- Environments such as LHCb VELO upgrade require all data to be sent off-detector, for each $25 \mathrm{~ns}$ bunch crossing $=>$ no time for triggers

- Several improvements in front end electronics

- Simultaneous timing and charge measurement

- Low expected timewalk $(<25 \mathrm{~ns})$

- Fast oscillator for timing measurements better than global clock frequency

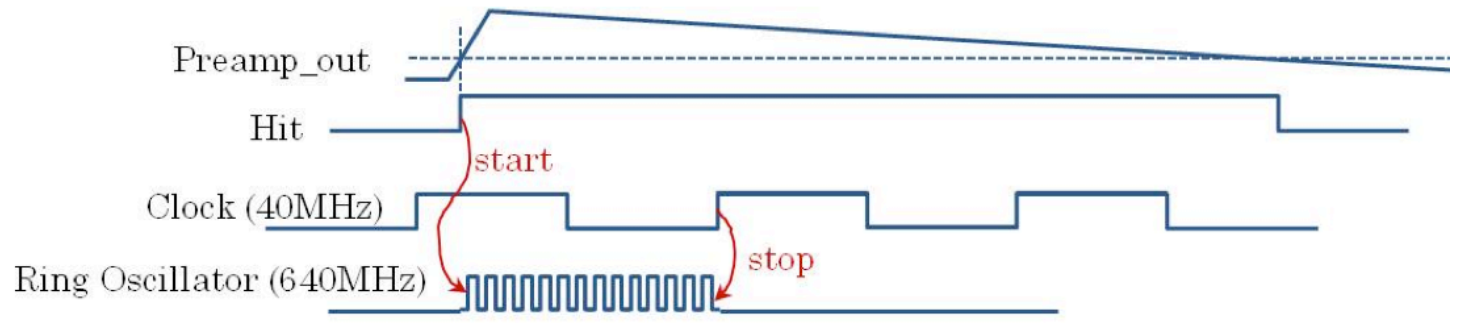




\section{TimePix3 telescope}

- Data scheme of TimePix3 telescope very different from current setup

- Data driven readout

- Up to $\sim 40 \mathrm{MHz}$ hit pixel rate ( $>10 \mathrm{MHz}$ raw beam)

- TimePix 3 timing resolution $(1.6 \mathrm{~ns}) \approx$ scintillator resolution

- Telescope would read out every particle, with dut recording hits at maximum sustainable rate (current beam intensities at CERN SPS $<1 \mathrm{MHz}$ average rate)

- Frame based readout (and associated pattern recognition) replaced with "clean" reconstruction environment

- Easy to distinguish noise clusters when tracking through several planes

- Low/zero combinatorics $=>$ fast processing time 